\title{
Study of Correlation between Magnetic Resonance Spectroscopy Metabolites and Glioma Grading
}

\author{
Rithi Melissa D'Silva ${ }^{1}$, Basavaraj N. Biradar ${ }^{2}$, H. B. Suresh ${ }^{3}$, Anston Vernon Braggs ${ }^{4}$, Mahesh ${ }^{5}$ \\ ${ }^{1}$ Resident, Department of Radiodiagnosis, ${ }^{2}$ Assistant Professor, Department of Radiodiagnosis, ${ }^{3}$ Professor, Head of \\ Department, Department of Radiodiagnosis, ${ }^{4}$ Senior Resident, Department of Radiodiagnosis, ${ }^{5}$ Senior Resident, Department \\ of Radiodiagnosis, Father Muller Medical College Hospital, India
}

Corresponding author: Dr Basavaraj N. Biradar, Assistant Professor, Department of Radiodiagnosis, Father Muller Medical College Hospital, Kankanady, Mangalore - 575002, India

DOI: http://dx.doi.org/10.21276/ijcmsr.2019.4.3.51

How to cite this article: Rithi Melissa D’Silva, Basavaraj N. Biradar, H. B. Suresh, Anston Vernon Braggs, Mahesh. Study of correlation between magnetic resonance spectroscopy metabolites and glioma grading. International Journal of Contemporary Medicine Surgery and Radiology. 2019;4(3):C230-C233.

\section{A B S T R A C T}

Introduction: Gliomas are among the most common primary neoplasms in children and adults. Management plan for gliomas greatly depends on their grade. Based on their grade, pre or post-surgical radiation or chemotherapy may be advocated. The objective of our study was to evaluate the role of Magnetic Resonance Spectroscopy in differentiating grades of glioma and to assess the relationship of brain metabolite ratios.

Material and methods: Magnetic Resonance Spectroscopy was performed in 27 patients with gliomas which were histologically proven. Brain metabolite ratios were calculated at intermediate / long Echo Time. Tumours were graded as low grade or high grade gliomas by experienced radiologist based on Magnetic resonance imaging and Magnetic Resonance Spectroscopy findings. Post-surgery, the histologic grade of glioma was correlated with that of imaging grade.

Results: Overall sensitivity, specificity, positive and negative predictive values for classifying glioma into grades using Magnetic Resonance Imaging and Magnetic Resonance Spectroscopy was found to be $83 \%, 66 \%, 66 \%$ and $83 \%$ respectively. Among the metabolite ratios, Choline-to- Creatine was found to be most useful in grading gliomas as low and high grade.

Conclusion: Magnetic Resonance Spectroscopy is a useful tool in diagnosing gliomas. Various metabolite ratios can be used in grading gliomas by considering the peri tumoral spread and nature of tumour composition. Further studies on Magnetic Resonance Spectroscopy metabolites are required to improve the sensitivity and specificity of the diagnosing tools in grading gliomas.

Keywords: Pre Surgical Glioma Grading, Magnetic Resonance Imaging, Metabolite ratios.

\section{INTRODUCTION}

Gliomas are among the most common primary neoplasms of brain across all age groups and histologically have varying grades from low to high. ${ }^{1}$ Gliomas are imaged on contrast enhanced Computed Tomography (CECT) and Magnetic Resonance Imaging (MRI) to obtain useful data required for arriving at a diagnosis and aiding neurosurgeons with information required for further management. It is of pivotal importance to grade Gliomas as low or high-grade malignancy, since the management of the two differ. Based on the grade, pre or post-surgical radiation or chemotherapy may be advocated. The gold standard for grading of glioma is histopathology. ${ }^{2}$

Many studies regarding the relationship between Magnetic Resonance Spectroscopy (MRS) and gliomas have been performed but usefulness of glioma grading using MRS is not clear due to unavailability of standard criteria for data analysis. ${ }^{3}$ Hence it is necessary to use imaging techniques like the MRS to provide additional information in this era of advances in radiological diagnosis. Though extensive studies have been done on spectroscopic metabolites like choline, creatine and N-Acetyl Aspartate (NAA), very few studies regarding usefulness of other available metabolites are established.

MRS is available in most of the institutions with $1.5 \mathrm{~T}$ MRI and in those institutions where perfusion imaging is not performed. In such situations, MRS being an advanced application can be utilized at its best for further characterization of the lesion. We conducted the present study to grade gliomas using conventional MRI and MRS. This was to assess the sensitivity of MRS in grading gliomas.

\section{MATERIAL AND METHODS}

This study was a validation study conducted retrospectively with data retrieval period of 18 months.

Patient selection: Pre-operative imaging studies of patients with untreated gliomas were considered.

Inclusion criteria: All patients with a histopathologic diagnosis of glioma who have undergone preoperative MR imaging with Spectroscopy in our hospital setup. 
Exclusion criteria: Patients in whom histopathology report is inconclusive or unavailable.

Previously treated case of glioma.

Ethical clearance for the study was obtained by the institution research and ethical committee. The study included a total of 27 patients of which 15 and 12 were male and female patients respectively. The study age group was between 7 and 72 years, with a mean age of 45 . All cases where pathologically proven cases after surgery. All included cases where evaluated using contrast enhanced MRI as well as MRS. Perfusion was not carried out in our study.

The sample size was determined considering a sensitivity of 96.4\% for grading of gliomas with Choline to Creatine ratio $(\mathrm{Cho} / \mathrm{Cr})$ and/or Lipid Lactate to creatine ratio $(\mathrm{LL} / \mathrm{Cr}$ ) ratios at both short and intermediate echo time according to a study on 3T H-MR Spectroscopy in Grading of Cerebral Gliomas: Comparison of Short and Intermediate Echo Time Sequences by J.H. Kim et $\mathrm{al}^{4}$ and the allowable error being $7 \%$.

The formula used for calculation is $n=Z \alpha^{2} p(1-p) / e^{2}$

Where $\mathrm{n}=$ sample size

$\mathrm{Z} \alpha=1.96$ at $95 \%$ confidence interval and $\mathrm{e}=$ allowable error All brain MRI have been acquired on $1.5 \mathrm{~T}$ superconducting MRI unit (PHILIPS ACHIEVA 16 channel system) with a dedicated head coil.

MRS was performed using multi voxel MR spectroscopy sequence in PHILPS ACHIEVA 1.5T MRI scanner with parameters as follows; Multivoxel, Matrix M x P - $22 \mathrm{x}$ 19, slice thickness - $15 \mathrm{~mm}$, with TR/TE of 2000/144ms. Various metabolite ratios available from the data were analysed. Histopathologic diagnosis with grading of glioma were obtained. Correlation between the grading of gliomas using metabolite ratios of MRS as well as MRI features and the histopathology grading of glioma was performed. Metabolite ratios which were analysed included N-Acetyl Aspartate to creatine ratio $(\mathrm{NAA} / \mathrm{Cr}), \mathrm{Cho} / \mathrm{Cr}$, Choline to $\mathrm{N}$-Acetyl Aspartate ratio (Cho/NAA) and myoinositol to creatine ratio $(\mathrm{mI} / \mathrm{Cr})$.

Interpretation: Both quantitative and qualitative data analysis was performed. In quantitative assessment, the mean values for normal $\mathrm{NAA} / \mathrm{Cr}, \mathrm{Cho} / \mathrm{Cr}$ and $\mathrm{Cho} / \mathrm{NAA}$ were considered to be $1.44,0.66$ and 1.9 respectively. ${ }^{5,6}$ Regions of clinical interest were the regions showing diffusion restriction and contrast enhancement and peritumoral area. Baseline values of normal brain parenchyma was also obtained.

Qualitative analysis was performed on post contrast images and was based on conventional MRI reporting system which considered lesion size, extent, contrast enhancement, mass effect, necrosis/ hemorrhage within the tumour and multifocality.

Imaging data were interpreted by experienced radiologists using a dedicated post-processing workstation.

According to previous World health organization classification, gliomas are graded from $1-4$. Grade 1 includes small subset of low-grade gliomas. Grade 2 includes low grade astrocytoma and oligodendrogliomas. Grade 3 includes anaplastic astrocytoma and oligodendrogliomas. Grade 4 includes Glioblastoma multiforme ${ }^{7}$

\section{STATISTICAL ANALYSIS}

Data was statistically analyzed using percentage, sensitivity, specificity, positive predictive value and negative predictive value.

\section{RESULTS}

Our study included 15 male and 12 female patients. Of the 27 patients, radiologically 12 were diagnosed to have low grade gliomas and 15 were diagnosed to have high grade gliomas (figure-1). Among these, pathologically 15 were proved to be low grade whereas 12 were proved to be high grade gliomas (Table 1). MRI and MRS features were applied to diagnose and grade gliomas preoperatively. Overall sensitivity, specificity, positive and negative predictive values for classifying glioma into grades using MRI and MRS was found to be $83 \%, 66 \%, 66 \%$ and $83 \%$ respectively (fig-2). Individual metabolite ratios were also assessed to classify gliomas into grades. Sensitivity, specificity, Positive and negative predictive values for NAA/ Cr was found to be $75 \%$, $66 \%, 64 \%$ and $76 \%$ respectively; for $\mathrm{Cho} / \mathrm{Cr}$ it was found to be $83 \%, 86 \%, 83 \%$ and $86 \%$ respectively and for Cho/NAA it was found to be $75 \%, 86 \%, 81 \%$ and $81 \%$ respectively. In our study $\mathrm{mIn} / \mathrm{Cr}$ did not show any significant variation in values with different grades of gliomas. We found that among all the metabolite ratios, $\mathrm{Cho} / \mathrm{Cr}$ had better statistical significance.

\begin{tabular}{|l|c|c|}
\hline Diagnosis & Frequency & Percentage (\%) \\
\hline Anaplastic oligodendroglioma & 6 & 22.2 \\
\hline Ganglioglioma & 4 & 14.8 \\
\hline Dnet & 1 & 3.7 \\
\hline Pilocytic astrocytoma & 5 & 18.5 \\
\hline Angiocentric glioma & 1 & 3.7 \\
\hline Anaplastic astrocytoma & 2 & 7.4 \\
\hline Atypical ganglioglioma & 1 & 3.7 \\
\hline Oligoastrocytoma & 2 & 7.4 \\
\hline Oligodendroglioma & 2 & 7.4 \\
\hline Meduloblastoma & 1 & 3.7 \\
\hline Glioblastoma & 2 & 7.4 \\
\hline Total Table-1: Histopathology diagnosis \\
\hline \multicolumn{2}{|c|}{27} & 100 \\
\hline
\end{tabular}

\section{DISCUSSION}

Gliomas are one of the most common primary cerebral tumours. 'Glioma' refers to tumours which are histologically similar to glial cells which include astrocytes, ependymal cells and oligodendrocytes. There have been various revisions for the classification of gliomas beginning from 1926. The modern classification system by World Health Organization (WHO) was first published in 1979 and has been revised since then with the latest fourth edition published in $2016 .{ }^{8}$ The histologic features considered in grading gliomas include cellularity, mitotic activity, nuclear atypia, vascularity and necrosis. Among the various cell types in gliomas, astrocytic gliomas are most common. In children low grade gliomas and in adults' high-grade diffuse gliomas are found to be more common. ${ }^{8}$

Among the conventional MRI sequences, T2 weighted 


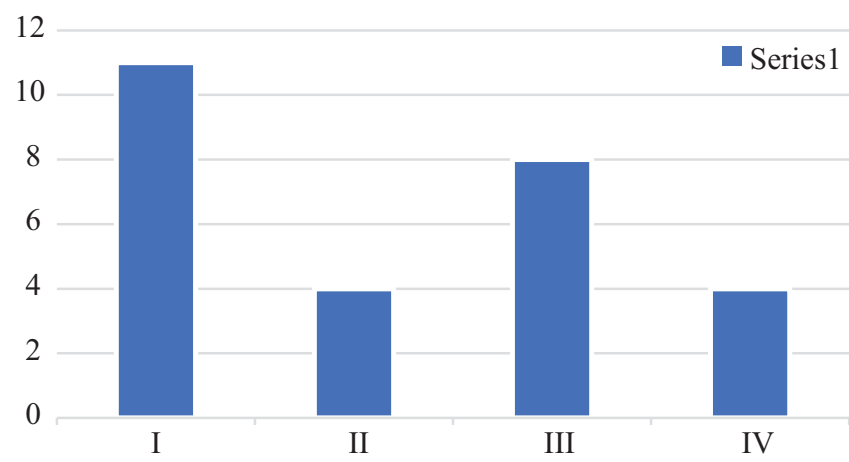

Figure-1: frequency of grades of glioma

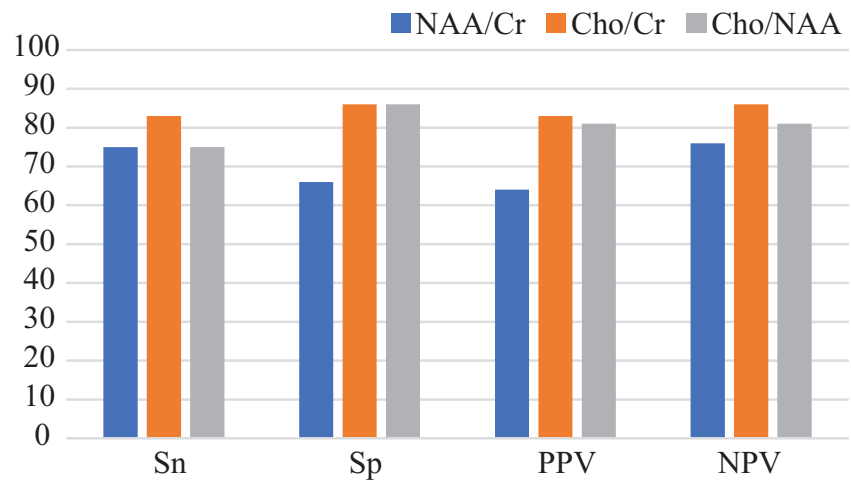

Figure-2: statistics of metabolite ratios in grading gliomas

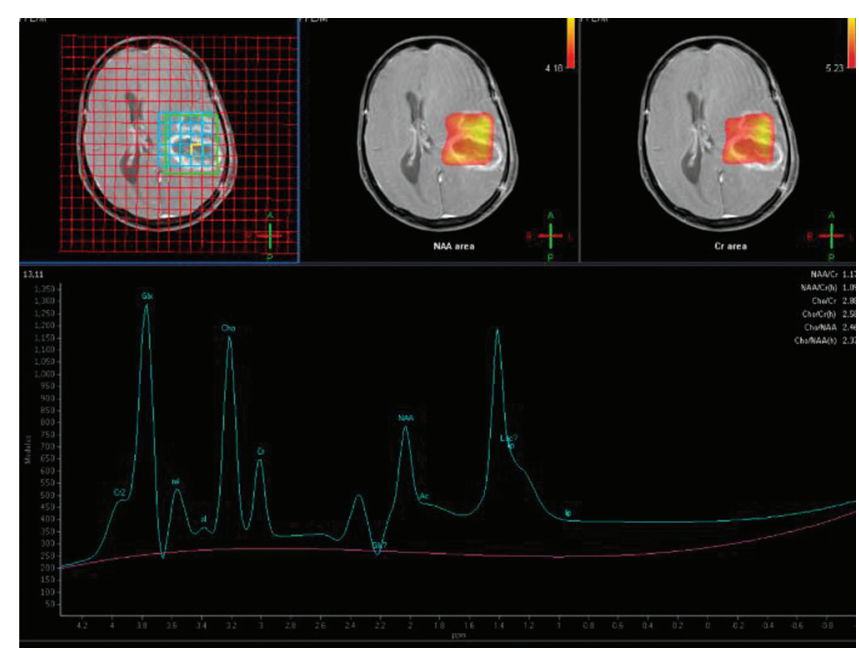

Figure-3: Multivoxel MR Spectroscopy in a contrast enhancing lesion demonstrates pathological brain metabolites spectrum with increased choline-to-creatine ratio and decreased NAA to creatine ratio suggestive of High grade glioma

images (T2W) and diffusion weighted images (DWI) can be used to evaluate the cellularity of a lesion. DWI helps to assess free water molecule diffusion. The same concept is used to assess the cellularity in gliomas. DWI is also used in diagnosis of acute cerebral infarction and in differentiating tumour necrosis from abscess cavity. Lower Apparent Diffusion Co efficient (ADC) values correspond to increased cellularity and hence high-grade gliomas. ${ }^{9}$

Tumour aggressiveness can also be assessed by peritumoral invasion. However conventional MR imaging sequences fail to evaluate this index of tumour aggressiveness. This is because of overlap between edema surrounding the tumour and tumour cell infiltration. Among the newer developments in MR sequences, Diffusion Tensor Imaging (DTI) is beneficial in this regard. MRS to some extent can also detect peritumoral infiltration by evaluating metabolites in the area surrounding the tumour region. ${ }^{9}$

Metabolite ratios have been used to assess cell proliferation and composition. Among the various metabolites that can be identified on MRS, the few metabolites which are of clinical significance in diagnosing gliomas include the following ${ }^{10}$ : N-Acetyl Aspartate (NAA) at $2.02 \mathrm{ppm}$, Choline (Ch) at $3.2 \mathrm{ppm}$, Creatine/ Phosphocreatine $(\mathrm{Cr})$ at $3.0 \mathrm{ppm}$, Lactate (Lac) at $1.33 \mathrm{ppm}$, Lipids (Lip) at 0.9-1.5 ppm and Myo-inositol (mI) at $3.56 \mathrm{ppm}$.

NAA is predominantly restricted to neurons, axons and dendrites and is referred to as neuronal marker. It acts as a marker of good neuronal health and is the largest signal in normal adult brain. Choline peak is a composite peak and the compounds involved in the peak are responsible for membrane synthesis and degradation. Hence choline peak is elevated in conditions where there is increased cell turnover like in tumours. Glial cells also have high choline levels. Creatine peak is also a composite peak and it represents energy metabolism generating ATP. Glial cells also have high creatine level compared to neurons (invitro). Myoinositol is mostly seen in short echo spectra. Glial cells are said to have higher levels of mIn than neuronal cells (invitro). ${ }^{11}$

Characteristically low-grade gliomas tend to show a relatively higher concentration of NAA and low levels of choline with lactates and lipids being absent. Earlier progression and malignant transformation of low-grade gliomas show increasing levels of creatine. Decreasing trend of NAA and myoinositol with progressive elevation in choline levels reflect progression of glioma grade. However, choline levels are seen to increase up to grade III. Presence of lactate and lipids indicate malignant transformation of glial tumours and is more often a feature of grade IV than grade III. ${ }^{10}$

In a study of multivoxel MRS, Tamiya et al. showed a positive correlation between $\mathrm{Cho} / \mathrm{Cr}$ ratio and $\mathrm{Ki}-67$ index while NAA/Cho ratio has a negative relationship with the index. ${ }^{10}$ In a study by Kim $\mathrm{JH}$ et al. ${ }^{4}$ which compared Short and Intermediate Echo Time Sequences in spectroscopy to grade gliomas, $\mathrm{Cho} / \mathrm{Cr}$ and $\mathrm{LL} / \mathrm{Cr}$ ratios at either $\mathrm{TE}$ were similarly useful in differentiating high-grade gliomas from low-grade gliomas but short TE was preferable since there was poor lactate inversion at intermediate TE. However, in our study, metabolite ratios were not assessed at short TE, but at intermediate/ long TE. Cho/Cr and Cho/NAA helped in differentiating high grade and low-grade gliomas, with high grade gliomas showing ratios $>2$ and low-grade gliomas showing ratios $<2$ (fig-3). This was in concordance with a study by Hamed SAI et al on Grading of Brain Tumours using MR Spectroscopy: Diagnostic value at Short and Long TE which showed similar ratios. ${ }^{5}$

Also study by Guzmán-De-Villoria JA et al. ${ }^{12}$ on comparing added value of advanced over conventional magnetic resonance imaging in grading gliomas and other primary brain tumours, high grade tumours showed high choline/ creatine at long TE which was in concordance with our study. 
Significant differences were found on spectroscopy between the high- and low-grade gliomas with $\mathrm{P}$ value $<0.05$ in a study by Yang $\mathrm{D}$ et $\mathrm{al}^{13}$ in a study comparing multivoxel $2 \mathrm{D}$ chemical-shift imaging proton MR spectroscopy, echoplanar perfusion and diffusion-weighted MRI. This study concluded that echoplanar diffusion, perfusion MRI and multivoxel proton MRS offer diagnostic information superior to conventional MRI, in the assessment of glioma grade.

Since our study was limited to only MRS, it did not include perfusion imaging and this parameter could not be analyzed which was a drawback of the study. However, with improvement in technology, newer software's can be further used in grading tumours and further studies are required to explore its usefulness.

\section{CONCLUSION}

MRS is a useful tool in diagnosing gliomas and other brain tumours. Various metabolite ratios can be used in grading gliomas by considering the peri tumoral spread and nature of tumour composition. Further studies on MRS metabolites are required in conjunction with other advanced MRI applications to improve the sensitivity and specificity of the diagnosing tools in grading gliomas.

\section{REFERENCES}

1. Zidan S, Hazim I. Tantawy, Makia MA. High grade gliomas: The role of dynamic contrast-enhanced susceptibility-weighted perfusion MRI and proton MR spectroscopic imaging in differentiating grade III from grade IV. 2016;47(4):1565-1573.

2. Wesseling P, Kros JM, Jeuken JWM. The pathological diagnosis of diffuse gliomas: towards a smart synthesis of microscopic and molecular information in a multidisciplinary context. Diagnostic histopathology. 2011;17(11):486-494.

3. Chernov MF, Ono Y,Muragaki Y,Kubo O,Nakamura R, Iseki $\mathrm{H}$ et al. Differentiation of High-Grade and LowGrade Gliomas Using Pattern Analysis of Long-Echo Single-Voxel Proton Magnetic Resonance Spectroscopy ((1)H-MRS). Neuroradiol J. 2008;21(3):338-49.

4. Kim JH, Chang KH, Na DG, Song IC, Kwon BJ, Han $\mathrm{MH}$ et al. 3T 1H-MR spectroscopy in grading of cerebral gliomas: comparison of short and intermediate echo time sequences. AJNR Am J Neuroradiol. 2006;27(7):1412-8.

5. Hamed SAI, Ayad CE. Grading of Brain Tumors using MR Spectroscopy: Diagnostic value at Short and Long TE. IOSR-JDMS. 2017;16(10):87-93.

6. Horská A, Barker PB. Imaging of brain tumors: MR spectroscopy and metabolic imaging. Neuroimaging Clin N Am. 2010;20(3):293-310.

7. Louis DN, Holland EC, Cairncross JG. Glioma classification: a molecular reappraisal. Am J Pathol 2001; 159(4):779.

8. Louis DN, Schiff D, Batchelor T. Classification and pathologic diagnosis of gliomas. Uptodate 2019. https://www.uptodate.com/contents/classification-andpathologic-diagnosis-of-gliomas.

9. Kao HW, Chiang SW, Chung HW, Tsai FY, Chen CY. Advanced MR imaging of gliomas: an update.Biomed
Res Int. 2013;2013:970586.

10. Bulik M, Jancalek R, Vanicek J, Skoch A, Mechl M. Potential of MR spectroscopy for assessment of glioma grading.Clin Neurol Neurosurg. 2013;115(2):146-53.

11. Zhu H, Barker PB. MR spectroscopy and spectroscopic imaging of the brain. Methods Mol Biol. 2011;711(5):203-26.

12. Guzmán-De-Villoria JA, Mateos-Pérez JM,FernándezGarcía P, Castro E, Desco M. Added value of advanced over conventional magnetic resonance imaging in grading gliomas and other primary brain tumors. Cancer Imaging. 2014;14(4):35.

13. Yang D,Korogi Y, Sugahara T, Kitajima M,Shigematsu Y, Liang L et al. Cerebral gliomas: prospective comparison of multivoxel $2 \mathrm{D}$ chemical-shift imaging proton $\mathrm{MR}$ spectroscopy, echoplanar perfusion and diffusionweighted MRI. Neuroradiology 2002;44(8):656-6.

\section{Source of Support: Nil; Conflict of Interest: None}

Submitted: 31-05-2019; Accepted: 24-06-2019; Published online: 21-09-2019 